EPJ Web of Conferences 60, 17002 (2013)

DOI: $10.1051 /$ epjconf $/ 20136017002$

(C) Owned by the authors, published by EDP Sciences, 2013

\title{
Distinguishing between lepton number violating scalars at the LHC
}

\author{
Francisco del Aguila $^{1, \mathrm{a}}$, Mikael Chala ${ }^{1, \mathrm{~b}}$, Arcadi Santamaria ${ }^{2, \mathrm{c}}$, and Jose Wudka ${ }^{3, \mathrm{~d}}$ \\ 1 CAFPE and Departamento de Física Teórica y del Cosmos, Universidad de Granada, E-18071 Granada, Spain \\ 2 Departament de Física Teòrica, Universitat de València and IFIC, Universitat de València-CSIC, Dr. Moliner 50, E-46100 \\ Burjassot (València), Spain \\ 3 Department of Physics and Astronomy, University of California, Riverside CA 92521-0413, USA
}

\begin{abstract}
Scalars with lepton number violating interactions decaying into lepton pairs, as those mediating the see-saw of type II, always include doubly-charged components. If these are observed at the LHC, their electroweak quantum numbers can be determined through their leptonic decays in pair and single production.
\end{abstract}

\section{Introduction}

LHC data, and especially the discovery of the Higgs boson, $[1,2]$ have confirmed the validity of the minimal Standard Model (SM) below few hundreds of $\mathrm{GeV}$, except for the very tiny neutrino masses. No other signal of new physics (NP) has been observed up to now, in general pushing the gap to the next NP scale beyond the TeV. Then, one may also wonder if the remaining SM predictions are fulfilled. In particular, at what extent the accidental symmetries of the SM, like lepton number (LN), are also exact, and if eventually the observation of their violation is within the LHC reach.

Although neutrino masses are so small, the mere observation of that they are non-vanishing requires the extension of the SM. Thus, if neutrinos are Dirac particles, we have to add their right-handed ( $\mathrm{RH})$ counterparts, leaving LN conserved; while if they are Majorana, new particles mediating LN violating (LNV) processes must be added. Which, if light enough, may be observable at the LHC [3] ${ }^{1}$. The simplest realization of this scenario results from the addition of heavy Majorana neutrinos, giving to the SM neutrinos a mass through the see-saw mechanism, named of type I [11-15]. The observation of these extra neutral leptons may be problematic at the LHC because in the absence of other interactions they are produced through their mixing with the SM leptons [16-18], which is bounded to be small [19]. Moreover, the corresponding SM backgrounds are also rather large at hadron colliders.

As a matter of fact, the less known sector within the $\mathrm{SM}$ is the scalar one; and if there is NP at the LHC reach, it is likely to be related with the Higgs sector. The most popular SM extension by the addition of new scalars vio-

\footnotetext{
a e-mail: faguila@ugr.es

b e-mail: miki@ugr.es

c e-mail: arcadi.santamaria@uv.es

d e-mail: jose.wudka@ucr.edu

1 In general, they also generate extra contributions to neutrinoless double $\beta$ decay, besides the standard one proportional to the electron neutrino Majorana mass; which are conveniently parametrized by the corresponding low energy effective operators [4-10].
}

lating $\mathrm{LN}$ is provided by the see-saw of type II, which gives neutrinos a Majorana mass through the exchange of an electro-weak scalar triplet of hypercharge $1,\left(\Delta^{++}, \Delta^{+}, \Delta^{0}\right)$, coupling to lepton doublet as well as to gauge boson pairs, and hence with no well-defined LN [20-22]. Stringent limits on the mass of $\Delta^{ \pm \pm}$in the $200-400 \mathrm{GeV}$ range have been already set by CMS [23] and ATLAS [24], assuming that this doubly-charged scalar mainly decays into two samesign leptons, and allowing for a variety of particular cases with fixed branching ratios into different lepton flavours ${ }^{2}$. The mass bounds on $m_{\Delta^{ \pm \pm}}$, however, depend strongly on the choice for these branching ratios. Moreover, in both analyses the decay rate into $W^{ \pm} W^{ \pm}$is assumed to be negligible, as are also the possible LNV signals.

Here we review the generalization of the type II seesaw scenario, where we consider the addition of scalar multiplets with a priori arbitrary isospin $T=0,1 / 2,1, \cdots$, and hypercharge $Y$, and no well-defined LN [27 ${ }^{3}$. In general, branching ratios into same-sign lepton and gauge boson pairs are also assumed to be sizable; and therefore, LNV eventually observable. It must be emphasized, however, that in the see-saw of type II, and in more elaborated models, both branching ratios are only similar in a small region of parameter space. Generally the doubly-charged scalars decay almost exclusively into same-sign lepton pairs; or alternatively into gauge bosons, though in this case the signal cannot be extracted from the background for the range of masses under consideration [36].

Our analysis has also a different purpose than the one guiding the discovery of a doubly-charged scalar $H^{ \pm \pm}$decaying into two like-charge leptons, and which has been carried out to derive present experimental limits on the corresponding cross-sections. Present bounds are obtained assuming a mass for $H^{ \pm \pm}$and some fixed branching ratios to

\footnotetext{
${ }^{2}$ In definite models as in the see-saw of type II, the Yukawa couplings giving neutrinos a mass are the same mediating the like-charge di-leptonic scalar decay, and they are then constrained $[25,26]$, but this is not so in general.

3 Models with isosinglets of hypercharge 2 have been proposed, for instance, in [28-31]; whereas the phenomenological implications of isodoublets with hypercharge $3 / 2$ are studied in [32-35].
} 
same-sign lepton pairs of a given flavor composition. Then, the comparison of the predicted number of events including SM backgrounds with the observed one provides the corresponding limit, as no departure from the SM prediction is observed. In contrast, we assume that the doublycharged scalar has been already observed to resonate in the same-sign di-lepton channel $l_{1}^{ \pm} l_{2}^{ \pm}$, with $l_{1,2}=e, \mu$, in which case we describe a process for measuring its branching ratios to $l l, l \tau, \tau \tau$ and $W W$, as well as its total pair production cross-section.

\section{Scalar production and decay}

Any doubly-charged scalar $H^{ \pm \pm}$showing a resonant behaviour in the invariant mass distribution of same-sign dileptons must couple to one of the two SM bilinears, $\overline{L_{L}^{c}} L_{L}$ and $\overline{l_{R}^{c}} l_{R},{ }^{4}$ with $\mathrm{LN}=2 .{ }^{5}$ There being no restriction on the hypercharge $Y$ and on the isospin $T$ of the electroweak multiplet it belongs to, except that one of its components must be doubly-charged: $|2-Y| \leq T$. Since we can write gauge invariant operators with this multiplet and any of the two $\mathrm{LN}=2$ lepton bilinears by including enough SM (charge-conjugated) Higgs doublets $\phi=\left(\phi^{+}, \phi^{0}\right)(\tilde{\phi}=$ $i \sigma_{2} \phi^{*}$, with $\sigma_{2}$ the second Pauli matrix). These in turn give rise to the effective coupling (decay) of the doublycharged scalar (in)to the two same-sign charged leptons, after electro-weak symmetry breaking when the Higgs acquires a vacuum expectation value $(\mathrm{VEV})<\phi^{0}>=v / \sqrt{2} \approx$ $174 \mathrm{GeV}$.

In contrast with its leptonic decay, which only depends on the size of the effective coupling of the corresponding operator and is then a priori a free parameter, the $H^{ \pm \pm}$production is fixed by gauge invariance and hence does depend on the electro-weak multiplet it belongs to, that is, on $T$ and $T_{3}^{H^{++}}=2-Y$.

\subsection{Doubly-charged scalar pair and single production}

At a hadron collider $H^{ \pm \pm}$are mainly pair produced through the $s$-channel exchange of photons and $Z$ bosons, being their electro-weak gauge couplings obtained from the corresponding kinetic term $\left(D_{\mu} H\right)^{\dagger}\left(D^{\mu} H\right)$. They can be also be produced singly through the s-channel exchange of $W$ bosons if $T \neq 0$. Both cross-sections are of electro-weak size but vary with the multiplet $H^{ \pm \pm}$belongs to [27]. For instance, they grow with $T$ for multiplets with components of charge at most $Q=T_{3}+Y=2$ (that is, with $T_{3}^{H^{++}}=T$ ). They can be also produced by vector boson fusion, which can be considered to be a next-order correction and will be neglected here ${ }^{6}$. Although the vector boson fusion contribution grows with the $H^{ \pm \pm}$mass, it is smaller than the one

\footnotetext{
${ }^{4} L_{L}^{c}=\left(v_{L}^{c}, l_{L}^{c}\right)$ is the SM lepton doublet with charge-conjugated fields, $\psi_{L}^{c}=\left(\psi_{L}\right)^{c}=C{\overline{\psi_{L}}}^{T}, \psi_{R}^{c}=\left(\psi_{R}\right)^{c}=C{\overline{\psi_{R}}}^{T}$.

5 The other combination $\frac{R}{L_{L}^{c}} l_{R}$ requires a $\gamma^{\mu}$ insertion because of the fermions' chirality, and hence the presence of a covariant derivative $D_{\mu}$ to make the operator Lorentz invariant. The corresponding operators are equivalent to the ones constructed with the other two bilinears through the use of the equations of motion.

${ }^{6}$ Single doubly-charged production through vector boson fusion can be eventually at the LHC reach for rather bizarre models
}

mediated by the s-channel electro-weak gauge boson exchange by almost an order of magnitude for multiplets satisfying $T_{3}^{H^{++}}=T$ and doubly-charged scalar masses below the $\mathrm{TeV}$; details will be provided in a forthcoming publication. In any case, the corresponding events have two extra jets with relatively large pseudo-rapidity which provide an extra handle to separate them.

Our purpose here is to review how to determine the doubly-charged scalar pair production cross-section, and thus measure $T_{3}^{H^{++}}$, using only four lepton final states. We concentrate for simplicity on the case $T_{3}^{H^{++}}=T,{ }^{7}$ thus restricting $T=0,1 / 2,1,3 / 2,2$.

\section{Sampling}

Large hadron colliders have become precision machines due to the excellent performance of the detectors and the high energy and luminosity reached. Thus, the understanding of the SM backgrounds seems to be well beyond initial expectations, and the LHC potential, for instance, for observing new resonances in leptonic channels is foreseen to be well beyond the $\mathrm{TeV}$ region in next LHC runs, although their properties can be only established for lower masses.

Here we review the example discussed in Ref. [27], where we assume a heavy scalar with a mass $m_{H^{ \pm \pm}}=500$ $\mathrm{GeV}$, belonging to a weak singlet $(T=0)$, doublet $(T=$ $1 / 2)$, triplet $(T=1)$, quadruplet $(T=3 / 2)$ or quintuplet $(T=2)$ of hypercharge $Y=2-T$, and a LHC center of mass energy $\sqrt{s}=14 \mathrm{TeV}$. Doubly-charged scalar pairs are then generated using MADGRAPH5 [44], after implementing the corresponding vertices for the different multiplet assignments (see Eqs. (3-4) in Ref. [27]) in Feynrules [45], and the CTEQ6L1 parton distribution functions. Backgrounds are evaluated with ALPGENV2.13 [46], while parton radiation and fragmentation are simulated with PYTHIAV6 [47] and the detector with DELPHESV1.9 [48]. In the analyses, leptons have a minimum transverse momentum $p_{T}^{l}>15 \mathrm{GeV}$ and a maximum pseudo-rapidity $\left|\eta_{l}\right|<2.5$.

\subsection{Pair production cross-section determination}

We are interested in measuring the total cross-section $\sigma$ in order to determine the isospin of the multiplet the doublycharged scalar belongs to. However, this cannot be done using a single decay mode because the corresponding number of signal events also depends on the $H^{ \pm \pm}$branching ratio into this mode, which is in principle unknown. Thus, we must determine the number of events for each channel and sum them appropriately taking into account the different efficiencies to estimate the total cross section. What can be done using only events with four charged leptons, properly

evading the stringent electro-weak constraints on the VEV of the neutral scalar partner of the doubly-charged scalar boson [37,38]. In this case the doubly-charged scalar only decays into $\mathrm{W}$ pairs, and then it does not resonate in the di-leptonic channel.

${ }^{7}$ Models also including scalars with larger electric charges can produce even more striking signals [39-41]. In any case, we assume that mass splittings within multiplets due to mixing with other scalars are small, and can be neglected $[42,43]$. 
Table 1. Main applied cuts.

\begin{tabular}{lccc}
\hline Variable & $l^{ \pm} l^{ \pm} l^{\mp} l^{\mp}$ & $l^{ \pm} l^{ \pm} l^{\mp} \tau^{\mp}$ & $l^{ \pm} l^{ \pm} \tau^{ \pm} \tau^{ \pm}\left(W^{ \pm} W^{ \pm}\right)$ \\
\hline \# of leptons & 4 & 4 & 4 \\
$\left|m_{l_{1}^{ \pm} l_{2}^{ \pm}}-m_{H^{ \pm \pm}}\right|$ & $\leq 40 \mathrm{GeV}$ & $\leq 40 \mathrm{GeV}$ & $\leq 40 \mathrm{GeV}$ \\
$\left|m_{l_{3}^{ \pm} l_{4}^{ \pm}}-m_{H^{ \pm \pm}}\right|$ & $\leq 40 \mathrm{GeV}$ & $\geq 40 \mathrm{GeV}$ & $\geq 40 \mathrm{GeV}$ \\
$\not p_{T}$ & not applied & $\geq 50 \mathrm{GeV}$ & $\geq 50 \mathrm{GeV}$ \\
$x_{3}$ & not applied & $\geq 0.8$ & $\leq 0.7$ \\
\hline
\end{tabular}

sampled, and Eq. (7) in Ref. [27] is to measure

$$
\sigma=\left(\sigma_{l l l l}+\frac{1}{2} \sum_{a \neq l l} \sigma_{l l a}\right)^{2} / \sigma_{l l l l},
$$

where $\sigma_{a b}=\left(2-\delta_{a b}\right) \sigma z_{a} z_{b}$ is the $H^{ \pm \pm}$pair production cross-section into $a b$, with $z_{a} \equiv \operatorname{Br}(H \rightarrow a)$ the $H$ branching ratio into $a$ and $\sum_{a=l l, l \tau, \tau \tau, W W} z_{a}=1$.

In practice we proceed as follows. We select events with four charged leptons of the first two families with zero total electric charge, plus possibly missing transverse momentum, and require that at least one same-sign pair $l_{1} l_{2}$ reconstructs the scalar mass within $\pm 40 \mathrm{GeV}$. (See Table 1 for a summary of the main cuts.) These events are then separated into three disjoint sets depending on the category associated to the other like-sign pair: $l l, l \tau$ and $\tau \tau+W W$. We denote by $l l$ those events resulting from the decay of the second doubly-charged scalar into two leptons of the first two families, and hence, those with the second like-sign lepton pair $l_{3} l_{4}$ also reconstructing the scalar mass within $\pm 40 \mathrm{GeV}$. For the remaining events we assume that $l_{3}$ and $l_{4}$ are products of semileptonic tau decays, and distribute the transverse missing momentum of the event between both tau leptons with the requirement that their momenta align along the momentum of the corresponding product charged-lepton momentum it decays to: $x p_{\tau}^{\mu}=p_{l}^{\mu}$, with $0<x<1$. Then, we identify the event with the second scalar decaying into a lepton of the first two families and a tau lepton, $l \tau$, as those fulfilling that the fraction momentum of the most energetic of the two leptons not reconstructing the scalar mass, which we name $l_{3},\left(x_{3}\right)$ is larger than 0.8 . The other events are classified as resulting from the decay of the second scalar into two tau leptons or two gauge bosons, $\tau \tau+W W$.

Figures 1, 2 and 3 prove the usefulness of the procedure. Indeed, Figure 1 shows the like-sign di-lepton invariant mass for the lepton pair which provides the poorest reconstruction of the doubly-charged scalar mass and for its four decay modes: $H^{ \pm \pm} \rightarrow l_{3}^{ \pm} l_{4}^{ \pm}, H^{ \pm \pm} \rightarrow l_{3}^{ \pm} \tau^{ \pm} \rightarrow l_{3}^{ \pm} l_{4}^{ \pm} \not D_{T}$, $H^{ \pm \pm} \rightarrow \tau^{ \pm} \tau^{ \pm} \rightarrow l_{3}^{ \pm} l_{4}^{ \pm} \not p_{T}$, and $H^{ \pm \pm} \rightarrow W^{ \pm} W^{ \pm} \rightarrow l_{3}^{ \pm} l_{4}^{ \pm} \not p_{T}$. The separation of the first sample is obtained simply requiring $m_{l_{3}^{ \pm} l_{4}^{ \pm}}$to be in between $m_{H^{ \pm \pm}} \pm 40 \mathrm{GeV}$. In Figure 2 we plot the momentum fraction assigned to the most energetic lepton of the second like-sign pair for the former four types of scalar decays. As can be observed, $x_{3} \geq 0.8$ provides a rather clean separation of the first two decay modes from the last two. The different distributions reflect the fact that the leading lepton tends to be the one which is not a decay product of a $\tau$ or a $W$, and thus with $x_{3} \sim 1$. In order to further reduce the contamination we also require $x_{3} \leq 0.7$ for $\tau \tau+W W$ events. Finally, Figure 3 makes it clear that separating $\tau \tau$ and $W W$ decays is rather inefficient. However, what further justifies not doing this at this

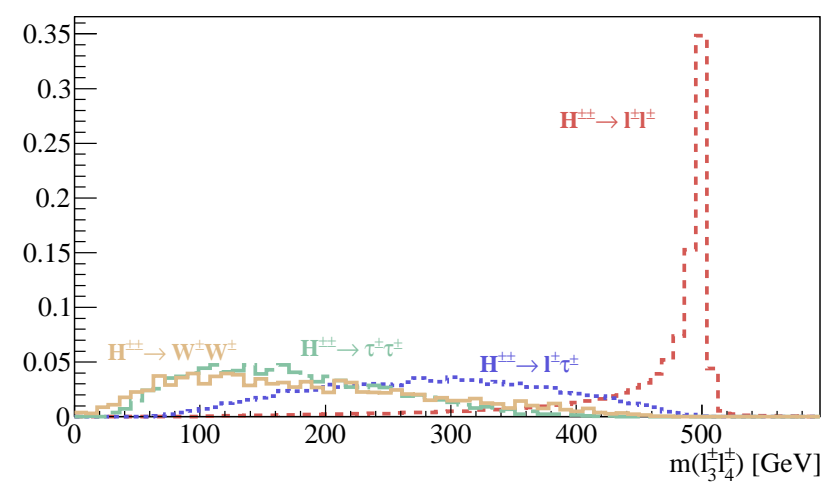

Fig. 1. Invariant mass of the two same-sign leptons that provides the poorest reconstruction of the $H^{ \pm \pm}$mass in doubly-charged scalar pair production for different decay modes.

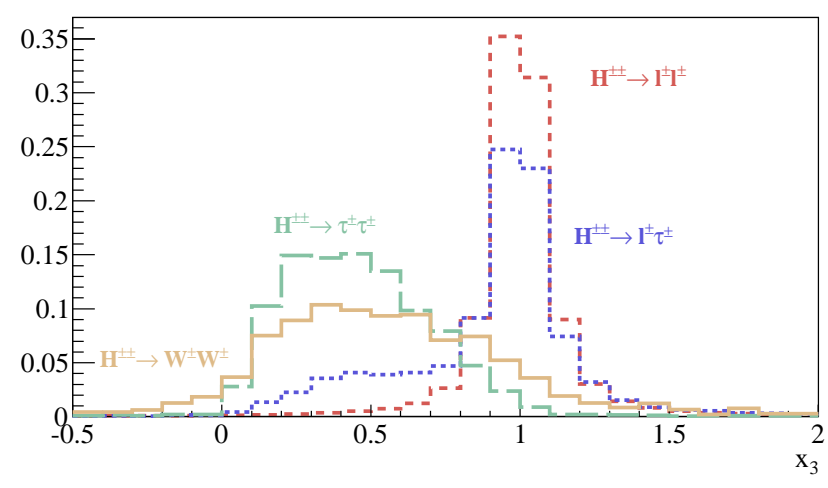

Fig. 2. Momentum fraction, $x_{3}$, of the most energetic lepton of the two which worst reconstruct the $H^{ \pm \pm}$mass in doubly-charged scalar pair production for different decay modes.

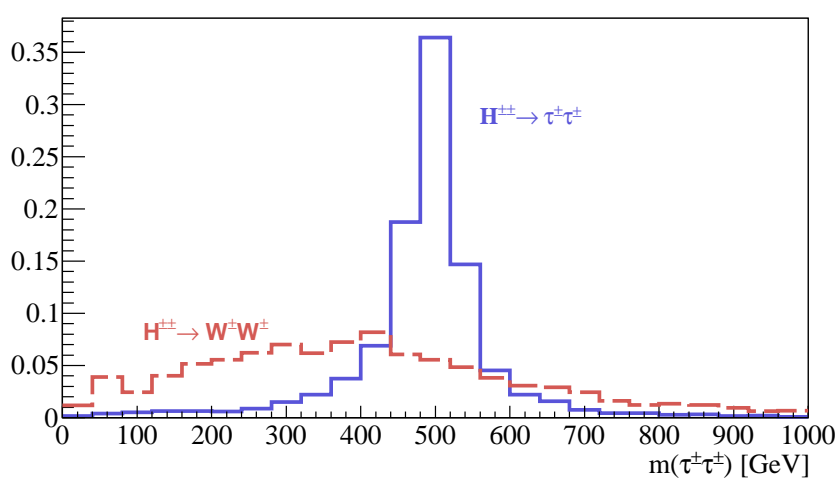

Fig. 3. Invariant mass distribution of the two reconstructed taus for both $l^{ \pm} l^{ \pm} W^{\mp} W^{\mp}$ and $l^{ \pm} l^{ \pm} \tau^{\mp} \tau^{\mp}$ decay channels in doublycharged scalar pair production.

stage, for evaluating the total pair production cross-section, is that the efficiency $\epsilon$ for evaluating the cross-sections of both types of processes is rather similar, allowing to treat both together consistently.

Actually, in order to measure the total pair production cross-section using Eq. (1), we have to estimate the efficiency for each process. From our Monte Carlo simulations we have calculated the efficiency for each subsample including the corresponding branching ratios, obtaining: $\epsilon_{l l l l}=0.6, \epsilon_{l l l \tau}=0.09$ and $\epsilon_{l l \tau \tau}=\epsilon_{l l W W}=0.02$, respectively. Then, counting the number of events of each of the 


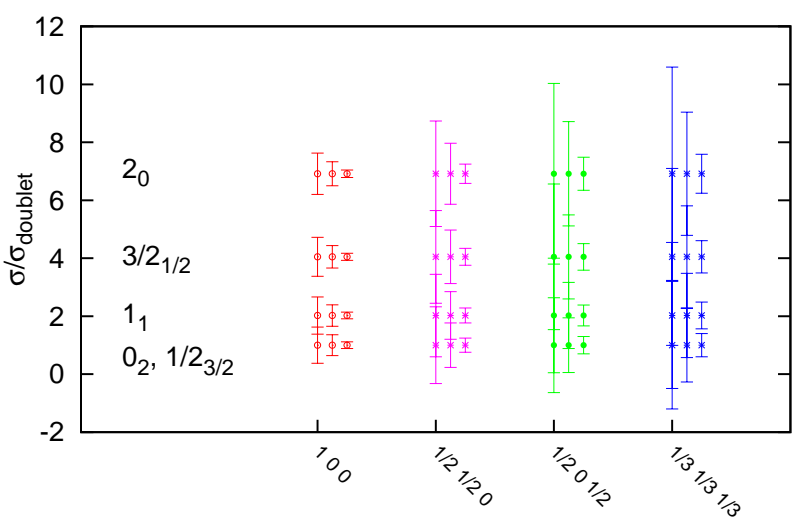

Fig. 4. Uncertainty on the determination of $\sigma\left(p p \rightarrow H^{++} H^{--}\right)$ for different $H^{ \pm \pm}$branching ratio assumptions at three different luminosities: 100, 300 and $3000 \mathrm{fb}^{-1}$. The labels on the left stand for the doubly-charged scalar $T_{Y}$ quantum numbers.

three subsets and dividing by the corresponding efficiency, we can measure the doubly-charged scalar pair production cross-section, once the integrated luminosity $\mathcal{L}$ is known. In Figure 4 we plot the statistical error for such a determination for several $H^{ \pm \pm}$branching ratio assumptions and the five multiplet assignments $T_{Y}$ discussed above. The crosssections are correctly reproduced because they are an input, but what matters is with which precision can we measure them in order to distinguish between different multiplet assignments. We draw statistical errors including the effect of SM backgrounds for three different integrated luminosities: 100, 300 and $3000 \mathrm{fb}^{-1}$. (For example, we find $\sim 50$ background events for $\mathcal{L}=300 \mathrm{fb}^{-1}$ with four charged leptons adding to zero total electric charge and a same-sign pair reconstructing the $H^{ \pm \pm}$mass within $40 \mathrm{GeV}$.) We fix four definite sets of doubly-charged scalar branching ratios: $\left(z_{l l}, z_{l \tau}, z_{\tau \tau}+z_{W W}\right)=(1,0,0),(1 / 2,1 / 2,0),(1 / 2,0,1 / 2)$, $(1 / 3,1 / 3,1 / 3)$, being higher the precision with $z_{l l}>z_{l \tau}>$ $z_{\tau \tau}+z_{W W}$. This means worsening when worse is our ability to reconstruct the $H^{ \pm \pm}$mass.

As can be observed in Figure 4, there is no apparent difference between the singlet and doublet cases because their neutral cross-sections are rather similar, but this is not so for the charged ones as there is no charged-boson exchange graph in the singlet case [27]. Thus, production of a single doubly-charged scalar can be used to discriminate between these two multiplets. If there is a significant excess of events compatible with $H^{ \pm \pm} H^{\mp}$, the singlet hypothesis will be automatically ruled out. However, to establish whether this is the case must be carefully assessed because the observation of only three leptons in the final state does not uniquely characterize this (charged-current) process. For instance, pair produced doubly-charged scalars decaying into $l^{ \pm} l^{ \pm} l^{\mp} \tau^{\mp}, l^{ \pm} l^{ \pm} \tau^{\mp} \tau^{\mp}$ or $l^{ \pm} l^{ \pm} W^{\mp} W^{\mp}$ can also produce only three leptons if one $\tau$ or $W$ decays hadronically. Therefore, we need to rely on extra variables in order to forbid these (neutral-current) contributions. Two discriminators appear to be most convenient: the missing transverse momentum, $\not \not_{T}$, which is larger in single $H^{ \pm \pm}$production than in $l^{ \pm} l^{ \pm} l^{\mp} \tau^{\mp}$ events with $\tau^{\mp}$ decaying hadronically, which allows to separate both contributions; and the transverse invariant mass of the opposite-sign lepton and the missing

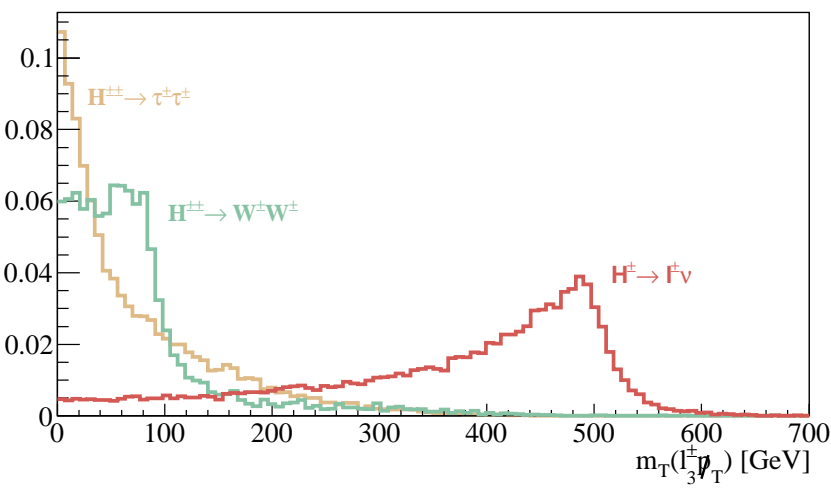

Fig. 5. Transverse mass distribution of the opposite-sign lepton and the missing tranverse momentum for several signals.

transverse momentun, $m_{T}\left(l_{3}^{ \pm} \not p_{T}\right)$, which peaks near the $H^{ \pm}$ mass in the charged-current process, as shown in Figure 5. It is apparent from this figure that a cut on $m_{T}>250 \mathrm{GeV}$ suppresses the neutral $\tau \tau$ and $W W$ contributions quite efficiently.

Although it is neither efficient nor necessary to separate $\tau \tau$ from $W W$ events in order to determine the total $H^{ \pm \pm}$ pair production cross-section in Eq. (1), one must attempt to do it in order to establish or not the violation of $\mathrm{LN}$ at the LHC. This separation makes use of the invariant mass distribution in Figure 3: $W W$ events are defined as those outside a wide enough interval around the $H^{ \pm \pm}$mass. Reconstructed $\tau \tau$ events near the doubly-charged scalar mass are interpreted as genuine $\tau \tau$ decays. For the events left, and interpreted as $W W$ decays, one must also check that the assignment is consistent with such a $H^{ \pm \pm}$decay. One can also check the consistency with the excess of events resulting from the semi-leptonic decay of the $W W$ pair, but this has other backgrounds, too, and will be discussed elsewhere.

\section{Acknowledgements}

This work has been supported in part by the Ministry of Economy and Competitiveness (MINECO), under the grant numbers FPA2006-05294, FPA2010-17915 and FPA201123897, by the Junta de Andalucía grants FQM 101 and FQM 6552, by the "Generalitat Valenciana" grant PROMETEO/2009/128, and by the U.S. Department of Energy grant No. DE-FG03-94ER40837. M.C. is supported by the MINECO under the FPU program.

\section{References}

1. G. Aad et al. [ATLAS Collaboration], Phys. Lett. B 716 (2012) 1 [arXiv:1207.7214 [hep-ex]].

2. S. Chatrchyan et al. [CMS Collaboration], Phys. Lett. B 716 (2012) 30 [arXiv:1207.7235 [hep-ex]].

3. W. -Y. Keung and G. Senjanovic, Phys. Rev. Lett. 50 (1983) 1427.

4. J. Schechter and J. W. F. Valle, Phys. Rev. D 25 (1982) 2951.

5. K. S. Babu and C. N. Leung, Nucl. Phys. B 619 (2001) 667 [hep-ph/0106054]. 
6. K. -w. Choi, K. S. Jeong and W. Y. Song, Phys. Rev. D 66 (2002) 093007 [hep-ph/0207180].

7. J. Engel and P. Vogel, Phys. Rev. C 69 (2004) 034304 [nucl-th/0311072].

8. A. de Gouvea and J. Jenkins, Phys. Rev. D 77 (2008) 013008 [arXiv:0708.1344 [hep-ph]].

9. F. del Aguila, A. Aparici, S. Bhattacharya, A. Santamaria, J. Wudka and, JHEP 1206 (2012) 146 [arXiv:1204.5986 [hep-ph]].

10. F. del Aguila, A. Aparici, S. Bhattacharya, A. Santamaria and J. Wudka, arXiv:1305.4900 [hep-ph].

11. P. Minkowski, Phys. Lett. B 67 (1977) 421.

12. T. Yanagida, Conf. Proc. C 7902131 (1979) 95.

13. M. Gell-Mann, P. Ramond and R. Slansky, Conf. Proc. C 790927 (1979) 315 [arXiv:1306.4669 [hep-th]].

14. S. L. Glashow, NATO Adv. Study Inst. Ser. B Phys. 59 (1980) 687.

15. R. N. Mohapatra and G. Senjanovic, Phys. Rev. Lett. 44 (1980) 912.

16. T. Han and B. Zhang, Phys. Rev. Lett. 97 (2006) 171804 [hep-ph/0604064].

17. F. del Aguila, J. A. Aguilar-Saavedra and R. Pittau, JHEP 0710 (2007) 047 [hep-ph/0703261].

18. A. Atre, T. Han, S. Pascoli and B. Zhang, JHEP 0905 (2009) 030 [arXiv:0901.3589 [hep-ph]].

19. F. del Aguila, J. de Blas and M. Perez-Victoria, Phys. Rev. D78 (2008) 013010 [arXiv:0803.4008 [hep-ph]].

20. R. E. Marshak and R. N. Mohapatra, In *Coral Gables 1980, Proceedings, Recent Developments In High-energy Physics*, 277-287

21. J. Schechter and J. W. F. Valle, Phys. Rev. D 22 (1980) 2227.

22. T. P. Cheng and L. -F. Li, Phys. Rev. D 22 (1980) 2860.

23. S. Chatrchyan et al. [CMS Collaboration], Eur. Phys. J. C 72 (2012) 2189 [arXiv:1207.2666 [hep-ex]].

24. G. Aad et al. [ATLAS Collaboration], Eur. Phys. J. C 72 (2012) 2244 [arXiv:1210.5070 [hep-ex]].

25. A. Hektor, M. Kadastik, M. Muntel, M. Raidal and L. Rebane, Nucl. Phys. B 787 (2007) 198 [arXiv:0705.1495 [hep-ph]].

26. F. del Aguila and J. A. Aguilar-Saavedra, Nucl. Phys. B813 (2009) 22-90, [arXiv:0808.2468 [hep-ph]].

27. F. del Aguila, M. Chala, A. Santamaria and J. Wudka, arXiv:1305.3904 [hep-ph].

28. A. Zee, Nucl. Phys. B 264 (1986) 99.

29. K. S. Babu, Phys. Lett. B 203 (1988) 132.

30. F. del Aguila, A. Aparici, S. Bhattacharya, A. Santamaria and J. Wudka, JHEP 1205 (2012) 133 [arXiv:1111.6960 [hep-ph]].

31. M. Gustafsson, J. M. No and M. A. Rivera, Approved for Phys.Rev.Lett.,2013 [arXiv:1212.4806 [hep-ph]].

32. J. F. Gunion, C. Loomis and K. T. Pitts, eConf C 960625 (1996) LTH096 [hep-ph/9610237].

33. V. Rentala, W. Shepherd and S. Su, Phys. Rev. D 84 (2011) 035004 [arXiv:1105.1379 [hep-ph]].

34. M. Aoki, S. Kanemura and K. Yagyu, Phys. Lett. B 702 (2011) 355 [Erratum-ibid. B 706 (2012) 495] [arXiv:1105.2075 [hep-ph]].

35. K. Yagyu, arXiv:1304.6338 [hep-ph].

36. S. Kanemura, K. Yagyu and H. Yokoya, arXiv:1305.2383 [hep-ph].

37. C. -W. Chiang, T. Nomura and K. Tsumura, Phys. Rev. D 85 (2012) 095023 [arXiv:1202.2014 [hep-ph]].
38. C. Englert, E. Re and M. Spannowsky, arXiv:1306.6228 [hep-ph].

39. K. S. Babu, S. Nandi and Z. Tavartkiladze, Phys. Rev. D 80 (2009) 071702 [arXiv:0905.2710 [hep-ph]].

40. G. Bambhaniya, J. Chakrabortty, S. Goswami and P. Konar, arXiv:1305.2795 [hep-ph].

41. R. Franceschini and R. N. Mohapatra, arXiv:1306.6108 [hep-ph].

42. A. G. Akeroyd and S. Moretti, Phys. Rev. D 84 (2011) 035028 [arXiv:1106.3427 [hep-ph]].

43. M. Aoki, S. Kanemura and K. Yagyu, Phys. Rev. D 85 (2012) 055007 [arXiv:1110.4625 [hep-ph]].

44. J. Alwall, M. Herquet, F. Maltoni, O. Mattelaer and T. Stelzer, JHEP 1106 (2011) 128 [arXiv:1106.0522 [hep-ph]].

45. N. D. Christensen and C. Duhr, Comput. Phys. Commun. 180 (2009) 1614 [arXiv:0806.4194 [hep-ph]].

46. M. L. Mangano, M. Moretti, F. Piccinini, R. Pittau and A. D. Polosa, JHEP 0307 (2003) 001 [hep-ph/0206293].

47. T. Sjostrand, S. Mrenna and P. Z. Skands, JHEP 0605 (2006) 026 [hep-ph/0603175].

48. S. Ovyn, X. Rouby and V. Lemaitre, arXiv:0903.2225 [hep-ph]. 